\title{
Design and Simulation of MAC Protocol for Vehicular Ad Hoc Network based on TDMA
}

\author{
AnLi $^{12}$ Ye Xuemei ${ }^{2}$ Fan Qinggang ${ }^{2}$ Zhang Hongmei ${ }^{1}$ \\ ${ }^{1}$ College of Science Air Force Engineering University, Xi'an 710038, China \\ ${ }^{2}$ Xi'an Research Institute of Hi-Tech Sian Shaanxi China 710025
}

\begin{abstract}
This paper proposes a new TDMA protocol- CL protocol to solve the problem of real-time data in 802.11 protocol cluster. It has a brand new frame structure and adopts the basis of carrier sense and backoff algorithm, which can solve the congestion problem. As can be verified by the final simulation modeling, it can effectively support transmission security of real-time business and has higher throughput.
\end{abstract}

Key words: vehicular ad hoc network; CL protocol; simulation; throughput

\section{Introduction}

VANET (Vehicular Ad Hoc Network) is a kind of ad hoc network which is dynamically formed through the use of wireless communication devices by vehicle nodes. Information transfer and sharing is realized through multi-hop packet forward between the vehicles, which makes it possible for the driver to obtain the information of real time traffic condition. As an important part of future ITS $^{[2]}$ (Intelligent Transportation Systems), VANET will provide communication services for a various of applications to improve the traffic efficiency and bring reliability, safety as well as convenience $[3,4]$
Compared with traditional Ad hoc network, it is characterized by nodes' high speed movement, more frequent change of topology, uneven distribution of nodes and so on ${ }^{[5]}$. As a result of VANET's large size and thousands of vehicles in network, communication congestions due to relatively large amount of communication in some areas of the network easily occurs, which will increase time delay and packet loss rate while lower route's performance.

In most of the recently MAC protocols, if there are more than one nodes that require to access channel in a short time interval, it will lead to random backoff and retransmission of many business. With continuous increase of the business volume, conflict cumulative will be caused, which will lead to the flood effect. Compared with random competition access, dynamic time slot allocation is more suitable to reduce the occurrence of conflicts. Therefore, a brand new MAC protocol of TDMA is designed-CL protocol.

\section{Analysis of CL protocol-MAC pro- tocol of TDMA vehicular Ad hoc network}

\subsection{Frame structure of MAC}

To solve hidden terminal problem in large scale multi-hop vehicular Ad hoc network, CL protocol adopts TDMA 
based dynamic time slot allocation protocol. Frame structure is consisted of control time slot, business time slot and access time slot. Transmission data is appointed during the Control time slot. Business time slot is used to transfer the data. Network access and synchronization problem is accomplished during the access time slot. Frame structure is illustrated in figure1:

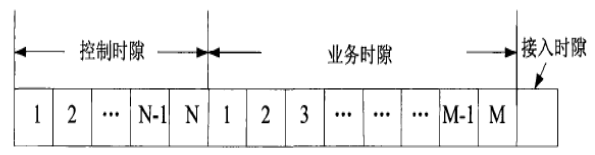

Fig1: frame structure of CL protocol

In figure 1, the previous $\mathrm{N}$ time slots are control frames and $\mathrm{N}$ is the total number of nodes in the network. Each node has a corresponding control time slot, where $1 、 2 \ldots \mathrm{N}-1 、 \mathrm{~N}$ are respectively the node number of the corresponding $\mathrm{N}$ nodes. The latter $\mathrm{M}$ time slots are business time slots. The last one is an access time slot.

The function of control time slot is that when the node needs to send data during its time slot, it should send reservation packet during the corresponding time slot. Whereas, after the data is sent by the node, it needs to send packets to inform its neighbor nodes during the control time slot and thus finishes the release process of corresponding business. This free business time slot can be used by other nodes, which will improve the use ratio of time slot.

In the Ad hoc, business time slots can be multiplexed in the space and there are a lot of free time slots in the channel. When the time reaches to the business time slot and the node state meets certain condition, they can use the same business time slot without conflicts. The number of business time slots $\mathrm{M}$ in this article is greater than the network number $\mathrm{N}$ and the nodes that have data to transmit can use multiple business time slots to convey data at a time.

After receiving synchronous information, the node firstly corrects local time reference. If there appear conflicts, it will conduct backoff using BEB algorithm and then access again. Otherwise, it will get a dedicated control channel after successfully accessed.

\subsection{Access method of the channel}

Synchronous mechanism adopts GPS timing mode. The access node must accomplish synchronization of GPS timing or can be only allowed to access the channel after receiving the synchronous information. When the synchronous information is received, this moment is taken as the initial moment for temporary time slot synchronization. Coarse adjustment of time slot is accomplished and access request to apply for accessing the network is sent by it. If the access time slot is a contention one and the node that is ready to access the network sends application to join the net at this time slot, there may be time slot contention problem. Nodes of the internal network send out frame synchronization information periodically. They will compute the value of signal delay according to the received access request signal and send it to the nodes that are ready to access by attaching it in the signaling. After receiving that information, the nodes will readjust reference time slot and thus accomplishes coarse time slot synchronization.

If access response signaling is received before access waiting timeout, the access is successful and finished. Or, it's considered that there occurs collision between the access request signaling. For this case, the node starts a random backoff counter. After a random period of time, it sends access request signaling again.

While effective frame synchronization information is detected, it judges and 
authenticates whether the launch node is a legal one or not. If not, this frame synchronization information packet will be discarded and it keeps on waiting. Otherwise, timeslot alignment is accomplished. Location information of time slot is subject to the moment frame synchronization information is received by the baseband.

After time slot alignment, it will send access request to initiating station. Access channel competitive preemption mechanism of Aloha is adopted. After application is precisely received by the initiating station, it first authenticates whether the applied access user is a legal one or not. If not, it sends reject information and instructs the user not to send access application information. When the node receives the reject information, it returns back to the starting state. Otherwise, the initiating station sends access application success and informs it to conduct latency adjustment. Access node will conduct coarse adjustment to time delay. Meanwhile, control number of home station is computed. What's more, frame synchronization information is sent and it allows the unconnected stations to access. If two nodes send access application to the same initiating station, initiating station will not receive access application information precisely. For this case, the node will launch access application after keeping off for sometime randomly.

\subsection{Distribution method of time slot}

In the Ad hoc network, the nodes between two hops are required to send data during same time slot, which can be realized interactively by controlling information of time slot. Greedy algorithm is adopted to distribute time slots. The wireless coverage area of each node in Ad hoc network is limited and the nodes can only perceive the information from their neighbor nodes. Greedy way is used to distribute time slots for the nodes, namely each node occupies the whole unused time slots followed by it.

\subsection{Node state}

To settle the hidden and exposed terminal problems of wireless Ad hoc network, five states are defined for the nodes: IDEL: channel is idle; TRANS: transmit status; RECEIVER: receive status; HIDDEN: node is a hidden terminal and sends silence status; EXPOSED: node is a exposed terminal.

All the nodes in the network judge whether they should receive and send information or keep off and wait. Therefore, state transfer list has wilder range of application here.

\section{Analysis of CL protocol simulation}

The network model is shown in figure 2 . The entire scene is $200000 \mathrm{~m} \times 200000 \mathrm{~m}$. The network is consisted of 24 mobile nodes. Omni antenna is used to broadcast and send messages. The wireless communication distance between two nodes is $3500 \mathrm{~m}$.

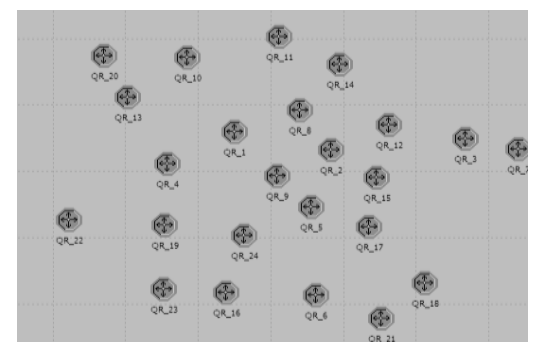

Fig2: simulation network model of CL protocol

Node model of the protocol is mainly consisted of data source module, sink module, upper interface module MAC_UP, QR module and wireless transceiver module, as is shown in figure 4 . Source module and sink module form the upper layer of MAC layer in Ad hoc network protocol stack. Source module chooses the simple source model of OP- 
NET software, which can set the properties directly such as packet length, time interval for packet generation, start time and end time for stack generation. To simulate channel environment in mixed business, the sources of the 24 nodes are set to be in different distribution models. Sink module is used to count or destroy the received packets.

\begin{tabular}{|ll|}
\hline Attribute & Yalue \\
\hline prome & source \\
- icon name & simple_source \\
Packet Format & processor \\
Packet Interarrival Time & QR_packet \\
-Packet Size & constant $(1.0)$ \\
-Start Time & constant (1024) \\
Stop Time & 10.0 \\
\hline
\end{tabular}

Fig3: source distribution set for QR protocol

MAC_UP module is the interface module between upper layer protocol and MAC layer, which can set destination MAC address for packets produced by upper layer. MAC_UP module that corresponds to information source in poisson distribution randomly chooses destination address inside its neighbor nodes according to uniform distribution. For the MAC_UP module corresponding to constant source, it chooses one node from its neighbor nodes as its destination address. Packets received by MAC layer from lower layer are submitted to sink module. The process files of QR protocol algorithm are included in $\mathrm{QR}$ module. Rt_0 and rr_$_{-} 0$ module are wireless transceivers.

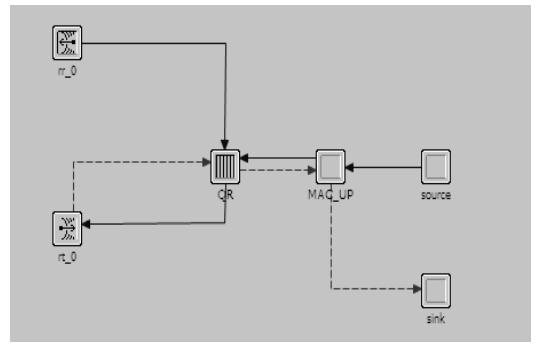

Fig4: node model of CL protocol

To simplify the analysis, propaga- tion time delay, guard space, synchronization time slot and processing delay are omitted in the simulation, which will not have too much influence on performance analysis. The selected simulation objects are respectively: network throughput $\mathrm{S}$ and access time delay of average packe $E(\tau)$. As improvements have been made in the distributed operation to reduce packet collisions and boost throughput, the key point of this simulation is the network throughput $\mathrm{S}$. To express the simulation result more accurately, here we choose the average statistics.

Table1: part of the simulation parameters for $\mathrm{CL}$ protocol

\begin{tabular}{llll}
\hline parameters & & parameters & \\
\hline $\begin{array}{l}\text { Channel } \\
\text { speed }\end{array}$ & $10 \mathrm{Mbit} / \mathrm{s}$ & $\begin{array}{l}\text { Move speed of } \\
\text { nodes }\end{array}$ & $15 \mathrm{~m} / \mathrm{s}$ \\
$\begin{array}{l}\text { Channel } \\
\text { number }\end{array}$ & 10 & $\begin{array}{l}\text { Node density of } \\
\text { one hop }\end{array}$ & $1 \sim 10$ \\
$\begin{array}{l}\text { Distribution } \\
\text { function of } \\
\text { source }\end{array}$ & $\begin{array}{l}\text { Uni- } \\
\text { form/poisson } \\
\text { distribution }\end{array}$ & $\begin{array}{l}\text { time slot } \\
\text { tion }\end{array}$ & $\begin{array}{c}\text { 300us } \\
\text { (chang }\end{array}$ \\
\hline
\end{tabular}

In order to get the performance of conflict elimination algorithm more precisely, the source of simulation node is set to be in parameter poisson distribution. And here we choose nodal load to be $G=5 \lambda \eta$. The figure is the simulation result of improved LC protocol's throughput $\mathrm{S}$ under different modal load $\lambda$. The objects used for reference and comparison are CL protocol and CSMA/CA protocol. To simplify the analysis process, here we adopt normalized network throughput $S=\lambda / N$, where $\lambda$ is the number of packets that are successfully interacted per second in the network under certain nodal load and $\mathrm{N}$ is the total packet number that source sends per second on the condition of node load with maximum throughput in the figure. To verify the guarantee CL protocol provided for real time business, we change the source sending distribution function of the nodes into poission and 
uniform distribution. Form figure 5, we can see that throughput will drop rapidly as the nodal load in CSMA/CA protocol reaches a certain value, which is the result of unsolved hidden terminal problem caused by random competition. Whereas, throughput can sustain a high level for $\mathrm{CL}$ protocol as load aggravates. The reason for this is that CL protocol is based on channel reservation and allocation and the node can access the channel successful via reservation.

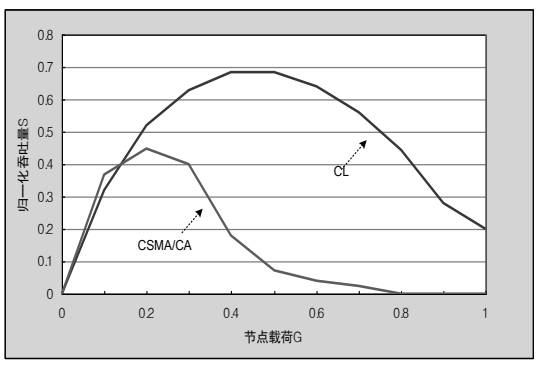

Fig5: normalized throughput under different nodal loads

Figure 6 is the simulation result of CL protocol's throughput $\mathrm{S}$ under different average packet lengths, where the value of APL is a multiple of 300 us. From the simulation result, we can see that CL protocol's throughput improves as with the increase of APL. This is reasonable as it can realize free conflict transmission in the data part. Therefore, large packet length can reduce competition time during unit interval and improve throughput.

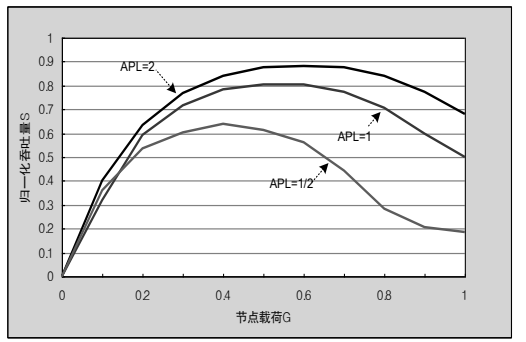

Fig6: normalized throughput under different APL conditions
Simulation result for average packet time delay on different nodal loads is shown in figure 7 . From the simulation result, it can be seen that average access time delays of CL business are all short and under $20 \mathrm{~ms}$ when the system is in light load $(\mathrm{G}<0.5)$. As the load aggravates, it can still keep relative short time delays through reservation. This is because that the nodes in CL protocol send data coordinately, which in one hand eliminates the packet conflict and in another hand realizes parallel transmission and improves transmission order of business. As can been seen, CL protocol still works effectively under heavy load $(\mathrm{G}>0.8)$. Compared with CL, competitions and conflicts increase doubly and time delay property became worse rapidly under heavy load condition in CSMA/CA protocol.

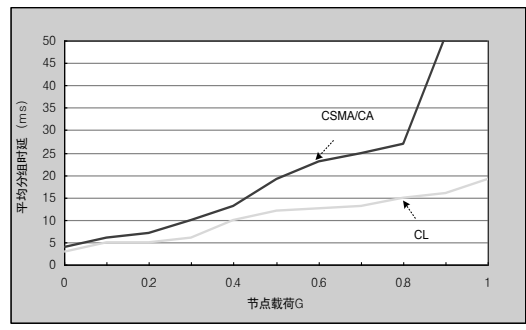

Figure 7: average packet time delay

\section{Conclusion}

This paper designs a dynamic time slot distribution protocol based on TDMA$\mathrm{CL}$ protocol. CL protocol has brand new frame structure and can solve the congestion problem. It can be verified by the simulation modeling that this protocol can effectively support the transmission security of real-time business and has higher throughput compared with CSMA/CA protocol. 


\section{Reference:}

[1] Karp B, Kung HT.GPSR: greedy perimeter stateless routing for wireless networks. Proceedings of the Sixth Annual ACM/IEEE International Mobile Computing and Networking.USA, 2000:243-254.

[2] Y Qian, N Moayeri. Design of Secure and Application-Oriented VANETS[C[. IEEE Vehicular Technology Conference, 2008: 2794-2799.

[3] Toyserkani AT, Strom EG, Svensson A. An efficient broadcast MAC scheme for traffic safety applications in automotive networks[J]. In: Proc. of the WCNC. Las Vegas: IEEE Press, 2006, 2100-2105

[4] Mimoza D, Arjan D, Leonard B. Emergency broadcast protocol for inter-vehicle communications. In: Proc. of the 11th Int'l Conference on Parallel and Distributed Systems. Fuduoka: IEEE Computer Society, 2005,.402406

[5] CAMP T, BOLENG J, DAV IES V.A survey of mobility models for Ad hoc network research. Wirless Communication and Mobile Computing, 2002, 2(5):483-502. 